\title{
An Analysis of Longitudinal Trends in Consumer Thoughts on Presence and Simulator Sickness in VR Games
}

\author{
John Porter III \\ Clemson University \\ Clemson, SC, USA \\ jjporte@clemson.edu
}

\author{
Andrew Robb \\ Clemson University \\ Clemson, SC, USA \\ arobb@clemson.edu
}

\begin{abstract}
Since the release of the Oculus Rift CV1 in 2016, millions of VR headsets have found their way into consumer homes. In this paper, we sought to understand what shifts have taken place within the two years since consumer VR became available. In this paper, we consider what can be learned about long-term use of consumer VR through an analysis of discussions in online forums devoted to VR. We gathered posts made on the $/ \mathrm{r} /$ Vive subreddit from the first two years after the HTC Vive's release. We present the results from an in-depth qualitative analysis concerning immersion, presence, and simulator sickness. Over time, as users moved from passive to active, their attitudes and expectations towards immersion and simulator sickness matured. Major trends of interest found were game design implementation and locomotion techniques.
\end{abstract}

\section{CCS Concepts}

-Human-centered computing $\rightarrow$ Virtual reality; Social content sharing; $\cdot$ Software and its engineering $\rightarrow$ Interactive games;

\section{Author Keywords}

Virtual Reality; VR Games; Immersion; Presence; Motion Sickness; Reddit;

\section{INTRODUCTION}

Since the release of the Oculus Rift CV1 in early 2016, virtual reality (VR) has rapidly transformed from a technology that was largely confined to the laboratory into a technology that is widely available in people's homes. Assuming VR continues its current growth and becomes entrenched within consumer society, we are currently at the beginning of a transitional period where users and developers are learning how to use this new technology. This period could be compared with the early era of smartphones, where users and companies tested and discarded many features (e.g. physical keyboards have largely disappeared from most cellphones, but were common in the early years of Android phones), or with the early era of film,

\footnotetext{
Permission to make digital or hard copies of all or part of this work for personal or classroom use is granted without fee provided that copies are not made or distributed for profit or commercial advantage and that copies bear this notice and the full citation on the first page. Copyrights for components of this work owned by others than the author(s) must be honored. Abstracting with credit is permitted. To copy otherwise, or republish, to post on servers or to redistribute to lists, requires prior specific permission and/or a fee. Request permissions from permissions@ acm.org.

CHI PLAY '19 October 22-25, 2019, Barcelona, Spain

(C) 2019 Copyright held by the owner/author(s). Publication rights licensed to ACM. ISBN 978-1-4503-6688-5/19/10 . \$15.00

DOI: $10.1145 / 3311350.3347159$
}

when film makers had to develop the 'vocabulary' of the pan, the cut, and the zoom, which we now view as commonplace.

In this paper, we sought to understand what shifts took place within the initial two years that consumer VR became available. To get an understanding of the broad trends in how people think about and use consumer VR, we turned to an analysis of discussions in online forums devoted to VR. This data represents a valuable opportunity to understand broad trends about actual usage in real settings, as opposed to the focused and controlled insights that can be gained in the laboratory. We chose to focus on two major topics of interest: presence (sometimes referred to as immersion) and simulator sickness. Presence is arguably one of the driving factors behind the use of VR for entertainment; the feeling of presence is the feeling of 'being' somewhere else in VR [22]. In contrast, simulator sickness is one of the major concerns that interferes with enjoyment of VR games, and limits what techniques can be employed when designing VR games [6, 19]. As such, we want to understand how users' beliefs, observations, and interests in these topics have evolved as users gained experience with them while playing VR games.

We gathered posts made on the /r/Vive subreddit, a community hosted on $w w w$.reddit.com that is focused on the HTC Vive HMD(Head Mounted Display). We chose to focus on this subreddit over others that are focused on competing headsets or general virtual reality, as /r/Vive was one of the more active communities in early 2016. The HTC Vive had also enabled the most compelling experience upon release due to the inclusion of motion controllers and a larger tracking volume. We gathered posts during the first two years after the HTC Vive's release, from April 2016 to April 2018. We sampled these posts at a 3 month interval for keywords related to our topics of interest. This data was then analyzed to extract themes in each month, which were then further analyzed to understand how these themes have evolved over time.

\section{LITERATURE REVIEW}

\section{Longitudinal Studies and Virtual Reality}

The goals of longitudinal research are to understand the "development of interacting processes over time" [10]. Aside from simulator sickness topics, little longitudinal research has been conducted in VR. Concerning this, our approach has been informed by published work in other related fields, in both descriptive and analytic terms [3, 7, 13, 9]. A number of longitudinal research studies have been conducted exploring consumer engagement with other forms of media, including 
video games [8, 2, 15, 1, 26, 4, 23], television [27, 12], smartphones [14, 11], and touchscreens [17]. Of these studies, research investigating engagement with video games is most relevant to consumer engagement with VR because of the similarities in content and their similar interactive nature. Several of these studies have evaluated natural behavior in online virtual worlds, such as Second Life [25] and World of Warcraft [20], and have examined a range of questions.

\section{Online Forums}

People often use online forums as a way to get answers to questions, tell stories, get access to expertise, offer their experiences, as well as read about the experiences of others [18]. Additionally, people have often sought advice from others using online discussion boards $[24,16]$. Concerning the most visited sites on the web, Reddit is currently ranked as \#6 in the United States, and \#21 globally to date ${ }^{1}$. The site describes itself as a "home to thousands of communities, endless conversation, and authentic human connection" where there are currently over 330 million active users, over 138 thousand active communities, and 14 billion monthly screenviews ${ }^{2}$. On Reddit, users can submit textual content directly as submissions, allowing for others to comment, as well as create their own subcommunities named "subreddits." These subreddits are independent, dedicated to a specific topic, and moderated by other volunteer 'Redditors' - a neologism combining 'Reddit' and 'editor'. Within these subreddits, users generally stay within their own community [5], and are often vetted by bots, moderators, and other redditors when posting content or answers in their respective forums. This signifies a generally cohesive and trustworthy base of information that can be gathered from these users. Currently, some longitudinal research concerning Reddit posts has been done, though only relating to Reddit data as a whole, not focusing on any one particular subreddit [21].

\section{METHODS}

For this research, we collected all posts made on the /r/Vive subreddit (subreddits are focused communities hosted on Reddit, a major user-driven website) for a two year period, starting on April 5th, 2016 (the day the HTC Vive was released). Conversations on reddit are structured around posts and comments, where a post is made by a user to start a conversation and comments are made within a post, either addressed to the original post or to a comment made by another user. This results in a more complex discussion structure than is common of most online forums. 121,550 posts and 2,183,924 comments were made on /r/Vive during the sampled period.

As our interest was how users' discussions about their experiences with the HTC Vive evolved over time, we chose to sample posts at a three month interval, where posts were sampled in month 0 (April 5th 2016 to May 4th 2016), month 3, month 6 , month 9 , month 12 , month 15 , month 18 , month 21, and month 24 (April 5th 2018 to May 4th 2018). Posts were collected for each month using the reddit API, via the portal hosted at $w w w$.redditsearch.io; the total number of posts

\footnotetext{
${ }_{1}$ https://www.alexa.com/siteinfo/reddit.com

${ }^{2}$ https://www.redditinc.com/
}

returned for each search term are shown in Figure 1. The searches for each date range were performed manually by one of the authors. We chose to focus at the post level, rather than the comment level. Thus, our search only returned results where the initial post contained the search term. All comments made to that post that were also relevant to the search term were also included in our analysis. If fewer than 40 posts were returned for the search period, each post and all of their comments were read. If more than 40 posts were returned for the search period, a sample of 40 posts were selected. This selection process was performed by one of the authors, with preference given to posts that appeared to discuss topics different from posts already selected for that month. We also attempted to select posts made during the entire time period, rather than only from a single portion of the time period.

We performed three keyword searches, one for 'immersion,' one for 'presence,' and one for 'motion sickness'. These search terms were selected after an initial exploratory search of terms related to presence and simulator sickness, our topics of interest in this paper. These three keywords were observed to be widely used by the community when discussing these topics of interest, stretching back to the time when the Vive was released. This observation is supported by Figure 1, which shows that these keywords remained in fairly constant use, proportional to the total number of posts made in each period analyzed. While the topics of presence and simulator sickness are both very distinct phenomenon, they are also essential to user experience in VR games. Presence being one of many major factors that drive player interest in VR games, and sickness being a major deterrent. As such, we chose to investigate how user discussions of each of these factors have evolved over time together.

Each search was conducted sequentially, beginning with 'motion sickness,' then 'immersion,' and finally 'presence'. Although initially we performed separate searches for 'immersion' and 'presence,' the results from the two were later merged due to their strong similarity. To analyze the data, all posts were read for a given month, and then re-read to extract relevant quotes or notes. Once each month was processed, we then categorized the collected data into various themes at the month level. Finally, major themes were extracted across all months. As previously mentioned, upon completion of both the 'immersion' and 'presence' analyses, we concluded that the themes identified for 'presence' mapped almost entirely onto the themes identified for 'immersion'. As there were few differences between people's use of the terms, we felt that it would simplify discussion to collapse similar topics found in both presence and immersion into one. If a topic was not mirrored for both keywords, then it was retained as a separate topic in the new 'presence/immersion' category.

\section{QUALITATIVE ANALYSIS}

\section{Presence and Immersion}

We identified 3 subtopics within the 'immersion/presence' keyword searches: Immersion Amplification focused on the hardware and design factors many users believed help amplify their feelings of immersion; Immersion Impediment focused on the opposite, hardware and design factors that impeded 


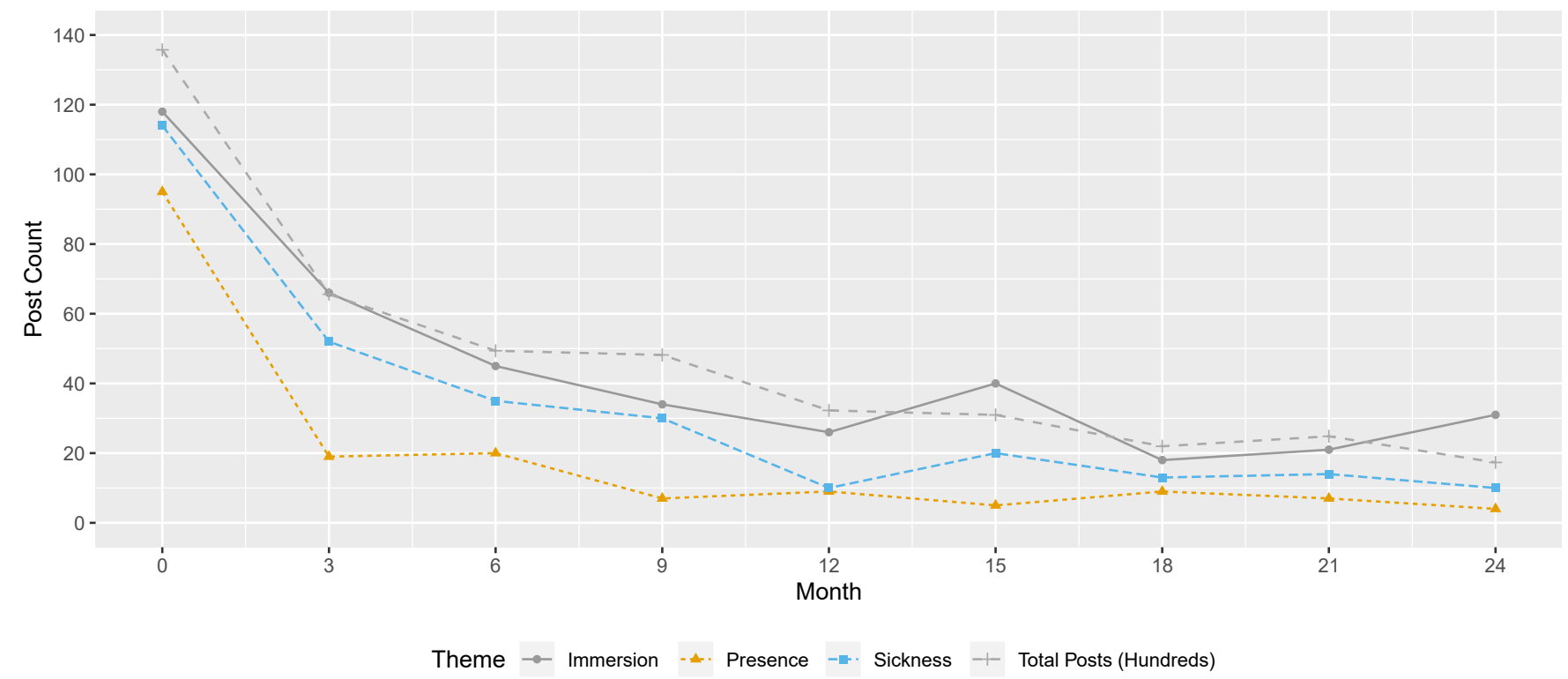

Figure 1. The plot shows total posts returned for each search term, by month. It also reports the total number of posts made in each month (in hundreds). While the number of posts containing our keywords decline over time, it can be seen that this decline is representative of a decrease in the number of total posts, not decreased interest in these topics.

feelings of immersion; and Locomotion's Effect on Immersion focused on various locomotion techniques and their impact on users' feelings of immersion.

\section{Immersion Amplification Discussion}

During the two year period we examined, users discussed several factors that enhanced their feelings of immersion. From these factors, 4 sub themes were extracted regarding this sentiment: Hardware Sets the Stage for Immersion focused on the impact the Vive's natural affordances had on user immersion; Modifying Hardware and Software to Improve Immersion then expanded on how outside hardware and software had amplifying effects on immersion as well; Good Game Design is Essential for Immersion highlighted the various aspects users enjoyed seeing in their games; and, Social Aspects add to Immersion, where users focused on how social aspects come into play when experiencing immersion in games.

Hardware Sets the Stage for Immersion: In the first month after release, discussion of immersion was scattered and often focused on the raw experiences of immersion, rather than what helped reinforce it. By month 3, users were:

"convinced that roomscale + tracked controllers were half the experience of VR, not just a nice addition, and that without these things, they don't even want to call it VR"

While often praising the Vive on it's "vertical FOV, motion controls," "fantastic tracking, room scale and $90 \mathrm{~Hz}$ refresh rate," throughout the months, users slowly focused their thoughts more on specific items they believed amplify their immersive experiences beyond the hardware, still agreeing that even though "the initial novelty of VR had worn off, the immersion hadn't." By month 6, conversations highlighting the hardware began to fade and more game-centered discussions began to rise, where users who would sometimes blame the hardware were often met with comments such as "you are overthinking it. Let go and stop focusing on the headset. Focus on the game." From here on much of the discussion regarding this faded as focus shifted from the hardware of VR instead to its applications.

Modifying Hardware and Software to Improve Immersion: In month 3, users highlighted how the use of peripheral hardware "made a world of a difference." Many users mentioned how when using gaming wheels "the force feedback felt amazing, and coupled with a VR headset it's incredibly immersive." This sentiment continued into month 9 where users began to seek more outside manipulation, noting that "other elements like touch and smell can also be manipulated to enhance immersion" and how they would "like some [software] mods to help out with immersion and stuff." Users began to ask questions like "why does this game have no haptics?" and demanded "more and better vibrations" in their applications. By month 15, debates had broken out concerning how different interactive controls influenced immersion, where some users believed that having a "HOTAS [controller] was very helpful...as it's one of the most immersive VR experiences currently possible," while others said that a "steam controller could be just as immersive as any hotas." But during this same month, users had also now become acclimated to the Vive's affordances and grew anxious for new immersion amplifying tools. Refusing to wait for companies to decide on what tools would be best for them to feel more directly engaged, many decided they "would like to try and build [their own]." Peripheral developers were falling behind on the wants of gamers who felt that when playing their games, "[holding] the rifle 
vs imagining it makes all the difference" as it "would significantly increase the immersion and improve [their] vr feeling." In month 21, the release of the TPCast "tetherless VR" system made "everything feel so much more immersive." But after many discussions concerning its pricing and unreliability, many users sought to find their own way and "mounted [their cables] to the wall instead" creating a "pulley system [to] satisfy" their immersive needs. In the following months, users continued in this modifying role by swapping the lens in the HTC Vive with others they found made their experiences "SO MUCH better...focused and vibrant, [taking] the immersion factor through the roof." Many were astonished and "could not believe that any 'user hack' could be better than a manufactured/tested product." This lead more users to continue to tinker and modify their hardware even more. Many users had now swapped out the padding in their Vive headsets with slimmer ones, as it had become commonly known to "increase your FOV dramatically." This sentiment had also carried over into software as well, where many agreed that incorporating "weather, texture, lighting, mesh, and audio mods took [their] immersion to visceral levels."

\section{Good Game Design is Essential for Immersion:}

"Good gameplay causes better immersion, bad gameplay hurts immersion; not the other way around"

This comment captures how users' often felt about the impact good game design has on enhancing feelings of immersion. Starting at month 6, users highlighted how they "don't think graphics actually affects the feeling of [immersion] as much as gameplay, scripting, and design do." In month 12, users agreed that "audio got less props than it should," and that "both sound fx and music made a huge difference in how a game 'feels' to play" as "it's often the sound more than the visuals that triggers a response from players." Level design was also an important mark of discussion, where one user highlighted one design that was so "spooky and atmospheric..that it made me feel like I was in another world with a real person." As time progressed, the role of interaction became prominent:

"it's not just the style of graphics, but the interaction...being able to do things that you both expect and surprise you"

Users often gave examples of specific forms of interaction that enhanced their immersion, frequently with reference to holding objects (e.g. "physically equipping your pickaxe") or saying that "using your hands makes immersion in the game so much more real." This sentiment only grew with the arrival of the Vive trackers which allowed users to "finally achieve full body movements." By the last month of our search, most users had by then become familiarized with immersion and utilized it as a metric to review games, (e.g. "Skyrim is awesome the world is great... but the immersion is OFF by A LOT," or how "swinging at the vein of ore to extract it...is such a fun, lovely little detail for immersion").

Social Aspects add to Immersion: From the early months users wanted social experiences involving other people, saying that "it always adds a level of immersion when there are other humans walking about the virtual environment" and that "seeing another player in there with you just adds SO much immersion and joy." Users were also concerned that the lack of many nonplayer characters (NPCs) in games was "something we don't get enough of in VR" because they "LOVE seeing the characters come in and talk to [them], it felt so... real...." By month 6 , users had actively experienced more social interactions in games and felt that "multiplayer gave [them] immersion, because [they] focus so much on being social that [they] lose that focus of it all being fake." This sentiment had grown by month 9 and users noted how their feelings of immersion were amplified when these social aspects were integrated well into the environment around them. For example, one user recounted how:

"One of the moments that hit me the hardest was when I entered a space port and entered my assign docking bay and looked up to see another player in a much more massive ship cruise over head and begin his own docking sequence. There is just something about the experience in VR, of being in a world of that scale while encountering another player who is in your same world and carrying out his own business"

By month 15, more games had successfully incorporated many of these ideals and users discussion shifted focus back to NPCs, where many still agreed that "having NPCs walking around...adds to the immersion by giving it a social feel." From here discussion fades concerning social topics until month 24, where many users by then had praised developers for finally taking NPCs "so far beyond what one can get playing the flat version" and how "[they] actually feel like [they're] being talked to, like really being talked to instead of just an NPC reading a script."

\section{Immersion Impediment Discussion}

Although users were able to find all the things they enjoyed about immersive VR, their experiences were also impacted negatively due to mirrored concerns. These concerns were condensed into 3 major sub themes: Users are not Entirely Satisfied with the Hardware focused on how users still have some issues regarding the Vive's shortcomings; while Improper Game Design and Affordance Implementation Hurts Immersion highlighted sentiment on gaming aspects that often held users back from achieving a fully immersive experience; but in Users Can Hurt their own Immersion we also found that the user themselves often assisted in their own immersive impedance.

Users are not Entirely Satisfied with the Hardware: Upon release, users complained about the many hardware issues they were experiencing, such as missing frames, flickering, and dead pixels. This lead to some backlash on the Vive and many follow-up discussions concerning return policies. Users also complained about how they felt when "the cable tension snapped your head back, as this is INCREDIBLY frustrating and sucks your immersion right out." By month 6, most users had begun analyzing the hardware, highlighting how the Vive's "Godrays are annoying" and as a result many "didn't feel a lot of immersion in the Vive because of the Fresnel lens artifacts." But discussion regarding these sentiments quickly faded as users began to "only notice it if [they] looked at it" and that 
eventually while "in games [they] were so involved with other stuff that [they] didn't think about it." No major discussions regarding these issues persisted after month 6 , although small isolated comments would sporadically arise in the months to come.

Improper Game Design and Affordance Implementation Hurts Immersion: Early month discussion regarding this impact occurred because users noted that many "games' physics were terrible," and that although many users were:

"able to accept a cartoon world, [but] if the physics was really off it was strange... and made it so [they] were not as invested in what's going on in the world"

One exemplar of this instance, was in month 6, when one user stated how:

"immersion-breaking [it was] to pick up a bucket with an object in it, only to watch that object clip through the bottom of the container and fall to the floor"

At this point, users demanded more from developers in regards to proper in-game affordance implementation, wanting more simplicity in some aspects (e.g. discerning which "buttons you cannot press" or what "small objects that you cannot lift"), and more complexity in others (e.g. wanting the game to not simply "ready an arrow in my hands, [but instead] make me draw it from a quiver.") In month 9 developers were warranted to "avoid including extremely extraneous or tedious actions for the sake of realism" because while "interactivity is important, it's not how much you have, so much as how you go about it." Meaning that while users did care about some elements of the visuals, they were much more focused on the implementation rather than visual style. This was noted when users felt that in some games "there was zero immersion because of how bad the scale was" and how seeing "the jagged edges" or "the horrible aliasing" in others broke their immersion. By month 12, most users had become fully accustomed to using motion control affordances in their games and had come to expect it. For most, a game having "no motion controls was a deal breaker...This kills the immersion" and that for some a game "COULD have been a blast, if they just added motion controller support." At this point, users had now become much more assertive in their understanding of what they wanted to see in their games, constantly hinting to developers in the forums that "room scale is extremely immersive, as long as everything is within reach," and to make sure that certain in-game components "translated well into VR." They also mentioned that when developing to consider how:

"locomotion is a crucial aspect of the gaming experience...[and that] having the 'wrong' kind for you can kill immersion or make you sick"

Users Can Hurt their own Immersion: Not all discussion of impedance is on the burden of the developer. A common theme among users, especially early in the Vive's release, was how users would oftentimes be the cause of their own impedance. The most common being when users were "experiencing some major tracking issues" and "it turned out the room setup was the problem." There were also many recorded HMD-related issues in the early months, where users realized their "headset wasn't perfectly adjusted," or their IPD was not taken into consideration because upon arrival, their Vive "had the lenses adjusted to half way" and they had not taken the proper steps to customize them. But for most, their issues could be solved simply by going "through room-setup again, [where] the immersion kicked in almost immediately" for them afterwards. These postings lasted until month 6 when we noted a drastic reduction of discussion regarding these issues. By month 9, these 'self-made error' discussions had transformed into advice columns where budding new users were brought up to speed on much of the knowledge previously discussed in the earlier month postings (e.g. how "setting [your Vive] up properly is crucial" and "re-centering the tracking is the cure" to most disruptions users experience with immersion).

\section{Locomotion's Effect on Immersion Discussion}

From early on, most users typically agreed that:

“teleportation does not aid in immersion, it's not a realistic or natural form of movement, but due to the current limitations in VR, it's detriment to immersion is offset by its ability to keep you from hurling your guts out"

Some users still refused to "support teleport-only games," while other users agreed that "if teleporting was really that central to your game design... [you should just] force everyone to teleport." This lasted until month 9, when the incorporation of trackpad locomotion (also known as smooth locomotion, where users navigated through virtual worlds as you would in a traditional console game via a trackpad or thumbstick) in games had become more prominent in discussion. This had little impact on the community however, as users thought that "magically sliding around a world with a trackpad was still no less immersive than pointing and teleporting." But at this point most users had compromised, agreeing that "forcing either type of locomotion...is bad for everyone" and that "it's nice to have both options available," so "offering both options is the best way to go." As a result, much of the earlier locomotive debates died down extensively by month 12 and many users agreed that "the answer is [for developers] to support multiple types of locomotion in a game." One user even stated that:

"at least 3 movement options should be available in my opinion...[and developers should] let the player choose what they are most comfortable with"

During months 15 and 18, we noticed that users had become more aggressive in their demands for more immersive locomotion techniques from developers. Many highlighted how teleportation was "rather immersion breaking," and how trackpad locomotion "gave some people simulator sickness." Although by this point "most games seemed to provide options, which is definitely the best way to go," even if discussion over preferences still remained a somewhat "polarizing issue." At the 2 year mark, two new community driven techniques had just arrived, Natural locomotion, and Freedom Locomotion. Natural locomotion was met with mixed response, where some felt it "made the immersion experience so much better...somehow just swinging my arms to move around gave me a sense of real physical movement." Others believed that "arm walking 
felt very unnatural" and it "did not seem like it provided me enough control compared to a joystick for movement." But because Natural Locomotion was a plug-in, many users could seamlessly add it into their games, even combining it with other locomotive techniques to get the most out of each, which helped to increase its acceptance as a viable addition. Freedom Locomotion, on the other hand, was very popular amongst users because it "felt real and natural and was more diverse in all the motions it integrated." But, due to it needing to be directly incorporated into games by developers, its overall use and incorporation suffered as a result.

\section{Motion Sickness}

We identified 3 subtopics within the 'motion sickness' keyword search: General Advice highlighted the best communally agreed practices to avoid, treat, and overcome sickness; Causes of Sickness focused on debates regarding the impact that gaming elements had on creating sickness and limiting experiences; and, finally, Understanding Sickness went through the development of the community's theoretical understanding of simulator sickness over time and how it related to individuals.

\section{General Advice Discussion}

Upon release, when inquiring about sickness, a large amount of comments regarding safety advice for combating motion sickness formed immediately as many users remained ignorant to proper hardware setup such as running suitable GPUs, using the correct USB ports, and proper room setup. This often lead to many "creating issues for [themselves]...so much it was causing [them] to be motion sick." Users were often advised by each other to first look for hardware issues because "maintaining a highly responsive VR system reduced motion sickness." This included "checking to see if you're dropping frames," "being sure your PC can always output 90 FPS," and that if "you start seeing it glitch out [to] either close your eyes and let it adjust or take the headset off." At the earliest signs of sickness symptoms, users recommended each other to "hit the pause button and exit the game immediately." Many experienced users often advised new users to:

"stick mainly to games that utilized room-scale or teleportation, and avoid ones with standard FPS [also known as smooth] locomotion as it feels very unnatural, which [they] believe is the origin of the nausea"

To negate negative effects, medical remedies were also recommended, including taking Dramamine and eating different variations of ginger, which were seen as helpful combatants to motion sickness. In months 3 and 6 , focus shifted more to preventing simulator sickness before and while inside the environment, rather than just reacting to it. This encompassed a variety of topics including keeping yourself cool during the experience, mentally preparing before entering for the environment, keeping your center of gravity, and "tweaking your IPD and making sure the headset is not loosely fitted to your face." Users were also instructed to "deal with any reflections on the lighthouses that [could be] causing any stuttering or juddery-ness." Although, some users would often still "mess with settings," creating an environment that intensified the chances of experiencing "motion sickness...[that] was completely [their] fault." But over the course of the next 6 months, users discussed more active approaches to breaking past simulator sickness, such as "slowly acclimating by exposing yourself to it a bit at a time." To reduce sickness when using certain locomotion techniques, many users advised "freezing your body" in place or "squinting your eyes during the sliding movements" and other visually intense events. Others advised more general advice such as "activating the comfort options" and "FOV reduction" in game settings, "knowing your personal limits," and "taking breaks periodically." By now, the use of ginger and Dramamine had now become widely received in the community as "over the counter medications for motion sickness." For the remainder of the sample, veteran users now understood that "different types of locomotion have their own difficulty and require their own adjustment period" and continued to guide new users and beginners accordingly. Many advised them to avoid starting with more complicated locomotive techniques such as smooth locomotion, and to begin with games that use teleportation locomotion, as it has now been generally agreed by the community to be the softest and least sickness inducing of the techniques.

\section{Causes of Sickness Discussion}

Upon release, users began to feel the symptoms of simulator sickness while playing their games, and immediately debated on what could be causing it. Users debated whether certain ingame mechanics were the main culprit for inducing sickness, such as developers "moving the player's point of view without them moving their head," "having locked points of references," and how "there weren't many feedback or visual cues to let you know what is happening" in certain games. Others referenced how some users may simply just be inducing their own sickness, as result of improper setup, technical issues, and general irresponsibility. By month 3, users had "experimented and made locomotion method[s]" of their own in order to curb simulator sickness symptoms e.g. ("a semi-teleportation method. You pick where you want to go but instead of it being instant it moves you in a path to that spot"). At this point there was also a growing understanding that some users just possessed a natural ability to handle artificial locomotive methods better than others. Many agreed that "the teleportation mechanic is great for people prone to nausea, [but still] wished more developers would give players different options." However, others still concerned with the safety of the overall community rebelled, stating that since "[teleportation] helps people who suffer from motion sickness" and "you don't want to risk someone feeling slightly uncomfortable," that all games should just use teleportation as a means of travel. This continued until month 6 , where many games had only one means of locomotion, limiting access from those uncomfortable with certain techniques. Unsatisfied, the sentiment continued to grow where many believed that:

"there shouldn't be some games where touchpad users feel they aren't getting the best experience they could, and motion sickness prone users feel as though they just have to put up with artificial locomotion" 
More continued to urge developers to "just give us both options so everyone will be happy" because "people are different." By months 12 and 15, users had discovered "options for movement [they] hadn't found before" and "more than half the people that get motion sickness often, [were finding] options that reduced it considerably," many "overcoming their locomotion sickness by having shorter play sessions." Starting at month 18, more forms of locomotion were being added to commercial games, although some users were still uncomfortable with these forms and were still unable to experience certain games. As a result, users continued to offer suggestions to developers on how to improve and expand the current locomotive techniques to support a broader audience and persistently requested for multiple in-game locomotive options for users to choose from. By month 21, most users realized "just how varied people's triggers for VR sickness are" and were willing to sacrifice immersion for more stability and control in games to avoid symptoms. In the meantime, users continued to aggressively seek and craft new locomotion techniques, but now understood that not all techniques will tailor to the majority.

\section{Understanding Sickness Discussion}

In month 0 , users debated the difference between the terms 'motion sickness' and 'VR sickness' (simulator sickness) and why they are both being used regularly to describe similar symptoms. Many "consider them to be two different things" that "work with different triggers," where:

"VR sickness isn't related to regular motion sickness...one is feeling like you're moving but not seeing movement, the other is the opposite"

By month 3, additional sickness-related terms had begun to surface and vetted users continued to explain the differences and similarities. Additionally, three distinct groupings had formed based on how strongly users were generally affected by simulator sickness. The sentiment being that "some get sick, some don't, and some adapt," where some users have a very high natural resistance, others a low resistance, and the remainder possess a moderate resistance with the ability to increase their resistance over time. This increased resistance was known by the community as obtaining one's 'VR Legs,' which was described as being "like sealegs, but for VR." The idea being that:

"practice makes perfect, and VR can take some getting used to. Some have it easier, some have it a bit more difficult, but if you power through you should be able to get your VR legs after a while"

With the understanding that this did not hold true for everyone because "some people [will still] get motion sickness, no matter how much they 'train' themselves." As time progressed some users began to theorize the underlying reasons of why some people were more or less subject to 'VR sickness,' such as if "previous experience in gaming" or if playing any specific gaming genres may have had any influence. But generally most were waiting to see "if research could actually start narrowing down WHY some people can get vr legs and some can't." Many researched studies, practices, and theories that were known concerning 'VR sickness', such as the importance of the vestibular system $[6,19]$, were widely discussed among the forums. But because researchers were still unable to agree on any single major explanation, the classification of the 3 groupings of user resistance became widely accepted. As a direct result, the community safely concluded what was believed in the beginning, in that "some people get it, and some don't, it's as simple as that." Discussion regarding this topic then sharply declined until months 21 and 24, where new users would come in occasionally to ask questions regarding these issues and would be met with the knowledge previously mentioned.

\section{DISCUSSION}

\section{Users' Transition from Novice to Expert}

Throughout all posts in both forums, we identified a major shift in how users' addressed both their enjoyment and their concerns. In the early months users discussed a variety of topics, detailing situations they stumbled across while experiencing VR in the Vive for the first time. Users often sought out for advice and guidance from one another regarding issues they had come across regarding both hardware or software. And although many would actively voice their opinions leading to a rich amount of discussion and debate, there were still many who would cloud discussion with crude jokes and irrelevant opinions. But within a year's time, most discussions had matured in their language and conversation. Soon many users that were not providing valid input in the topics being discussed were becoming identified and ignored by the community. More active users continued to discuss VR vocabulary and theories, as well as create their own. Eventually topics began to narrow and were becoming more strictly monitored by those within the community. Communal understanding and practices had began to form and many had become veterans both within the forums, as well as in their matured understanding of VR. By the end of our sample, users were now actively addressing developers and demanding changes rather than passively hoping for them. Users had shifted from being merely consumers of VR, to creators and analyzers, in both hardware and software. We also began to notice a more active role of developers reaching back within the forums to get more feedback from the community.

\section{User's Influence over Game Design}

Throughout the months, we saw that sentiment and discussion regarding game design and implementation changed over time between both forum searches. Initially, users were excited about all the new games and demos they were exposed to. But as time went on, many became familiar with the various gaming mechanics and their effects on the user experience in regards to both immersion and sickness. As more users continued to share their experiences with one another, certain patterns and techniques soon arose. While users enjoyed their provided graphics and interactions, they eventually began to critique them. Many highlighted ways to improve them by making sure developers knew what they enjoyed, as well as what made them sick. By the halfway mark, user focus shifted from gaming visuals to interaction techniques. We believe this was due to the initial 'awe' of VR visuals wearing off, where users were now more concerned with how they could make 
their experiences feel more personal. This included providing a more diverse amount of locomotion techniques as well as more social implementations, both with other players as well as AI characters. Many soon began creating and sharing their own content regarding these things, as they often felt large scale industry was not keeping up with their demands. By the end, users had become critics and reviewers of immersion rather than consumers of it, using it as a metric to weigh and rate games they played. In regards to motion sickness, the focus of game design by now was based entirely on having a diverse amount of locomotive techniques in games.

\section{Evolution of Locomotion}

Over the span of the 24 month period, user consensus over locomotion evolved constantly.

During the earliest months, most games used the same limited set of locomotion options: teleportation, smooth locomotion, and real-world walking (within a limited space), with teleportation being the most common method. User discussion around these options quickly focused on how they affected two factors: immersion/presence and simulator sickness. While no consensus was reached, the most common opinion in this period was that while teleportation produced low levels of immersion, it was still the best method as it was safe and did not produce much simulator sickness.

But as time passed, users became increasingly anxious, pushing developers to create more locomotive techniques centered around more fluid and natural interactions. Within a year after release, many users took the initiative upon themselves and developed techniques to increase the pool of locomotive techniques.

Eventually many discussions regarding exemplar locomotive techniques had ceased, as more users agreed that every user is unique and there is no one-locomotive-fits-all model for gaming. The conversation instead had now shifted to if the locomotive technique being applied was appropriate to each game's overall style. This change marked a new transition where users were then taking a more active role in conversations concerning future game development, often demanding that developers consider implementing a diverse amount of in-game locomotive options. Over the remaining months, this sentiment became a standard metric and a major factor of discussion among users regarding whether or not certain games should be purchased.

\section{Limitations}

A limitation with this research is that we only used three keywords when searching for relevant posts: immersion, presence, and simulator sickness. We chose to limit our search to these three terms after a long period of observing the forum wherein we noted that the majority of posts relevant to these topics contained these keywords. While these terms may not have captured all relevant posts, they were in common use by the community and were present in most discussions we observed.

\section{CONCLUSION}

Consumers in VR matured in both their attitudes and their expectations regarding immersion and simulator sickness. Af- ter initial user disappointments wore off, many were able to focus on the more positive aspects of VR. Users moved from passively accepting shortcomings provided to them to actively fixing them, or demanding that they be fixed. At first, hardware played a major role in immersion and simulator sickness discussion, but over time, hardware concerns were either addressed or noted as non-essential. From here, software became the major focus of these discussions for consumer VR users. Consideration of a variety of locomotive techniques when designing applications is recommended when creating new games. Developers also are encouraged to continuously seek out the latest information regarding safe and intuitive game design, both professionally and communally. Game design is a crucial factor which, if not implemented effectively, could risk many users to become sick. Likewise, game reviews from influential people can sometimes be the defining factor of its acceptance or rejection, so following good interaction technique implementation is also recommended. Therefore, developers are recommended to actively seek user feedback while in the process of creating applications for VR consumers to make sure they are in line with these sentiments. Users of VR are becoming increasingly active in the general community, both as consumers and creators, and are demanding more from both researchers and developers, in both speed and accuracy.

\section{ACKNOWLEDGMENTS}

We would like to thank the developers of Reddit and the individuals who shared their VR experiences that made this work possible. This work was supported in part by NSF Grant \#1717937.

\section{REFERENCES}

[1] Craig A Anderson and Brad J Bushman. 2001. Effects of violent video games on aggressive behavior, aggressive cognition, aggressive affect, physiological arousal, and prosocial behavior: A meta-analytic review of the scientific literature. Psychological science 12, 5 (2001), 353-359.

[2] Craig A Anderson, Akira Sakamoto, Douglas A Gentile, Nobuko Ihori, Akiko Shibuya, Shintaro Yukawa, Mayumi Naito, and Kumiko Kobayashi. 2008. Longitudinal effects of violent video games on aggression in Japan and the United States. Pediatrics 122, 5 (2008), e1067-e1072.

[3] Anol Bhattacherjee and G Premkumar. 2004. Understanding changes in belief and attitude toward information technology usage: A theoretical model and longitudinal test. MIS quarterly (2004), 229-254.

[4] Tom Boellstorff, Bonnie Nardi, Celia Pearce, and Tina L Taylor. 2012. Ethnography and virtual worlds: A handbook of method. Princeton University Press.

[5] Cody Buntain and Jennifer Golbeck. 2014. Identifying social roles in reddit using network structure. In Proceedings of the 23rd international conference on world wide web. ACM, 615-620.

[6] Simon Davis, Keith Nesbitt, and Eugene Nalivaiko. 2014. A systematic review of cybersickness. In 
Proceedings of the 2014 Conference on Interactive Entertainment. ACM, 1-9.

[7] Jesse Fox, Dylan Arena, and Jeremy N Bailenson. 2009. Virtual reality: A survival guide for the social scientist. Journal of Media Psychology 21, 3 (2009), 95-113.

[8] Douglas A Gentile, Craig A Anderson, Shintaro Yukawa, Nobuko Ihori, Muniba Saleem, Lim Kam Ming, Akiko Shibuya, Albert K Liau, Angeline Khoo, Brad J Bushman, and others. 2009. The effects of prosocial video games on prosocial behaviors: International evidence from correlational, longitudinal, and experimental studies. Personality and Social Psychology Bulletin 35, 6 (2009), 752-763.

[9] Jens Gerken, Peter Bak, and Harald Reiterer. 2007. Longitudinal evaluation methods in human-computer studies and visual analytics. In InfoVis.

[10] Jennie K Grammer, Jennifer L Coffman, Peter A Ornstein, and Frederick J Morrison. 2013. Change over time: Conducting longitudinal studies of children's cognitive development. Journal of Cognition and Development 14, 4 (2013), 515-528.

[11] AA Hadar, D Eliraz, A Lazarovits, U Alyagon, and A Zangen. 2015. Using longitudinal exposure to causally link smartphone usage to changes in behavior, cognition and right prefrontal neural activity. Brain Stimulation: Basic, Translational, and Clinical Research in Neuromodulation 8, 2 (2015), 318.

[12] Robert J Hancox, Barry J Milne, and Richie Poulton. 2004. Association between child and adolescent television viewing and adult health: a longitudinal birth cohort study. The Lancet 364, 9430 (2004), 257-262.

[13] Valerie Mendoza and David G Novick. 2005. Usability over time. In Proceedings of the 23rd annual international conference on Design of communication: documenting \& designing for pervasive information. ACM, 151-158.

[14] Lei Meng, Shu Liu, and Aaron Striegel. 2014. Analyzing the longitudinal impact of proximity, location, and personality on smartphone usage. Computational Social Networks 1, 1 (2014), 6.

[15] Ingrid Möller and Barbara Krahé. 2009. Exposure to violent video games and aggression in German adolescents: A longitudinal analysis. Aggressive Behavior: Official Journal of the International Society for Research on Aggression 35, 1 (2009), 75-89.

[16] Janet M Morahan-Martin. 2004. How internet users find, evaluate, and use online health information: a cross-cultural review. CyberPsychology \& Behavior 7, 5 (2004), 497-510.
[17] David Nicholas, Paul Huntington, and Peter Williams. 2003. Three years of digital consumer health information: a longitudinal study of the touch screen health kiosk. Information processing \& management 39 , 3 (2003), 479-502.

[18] Blair Nonnecke, Dorine Andrews, and Jenny Preece. 2006. Non-public and public online community participation: Needs, attitudes and behavior. Electronic Commerce Research 6, 1 (2006), 7-20.

[19] Lisa Rebenitsch and Charles Owen. 2016. Review on cybersickness in applications and visual displays. Virtual Reality 20, 2 (2016), 101-125.

[20] Daniel Schultheiss. 2007. Long-term motivations to play MMOGs: A longitudinal study on motivations, experience and behavior.. In DiGRA Conference.

[21] Philipp Singer, Fabian Flöck, Clemens Meinhart, Elias Zeitfogel, and Markus Strohmaier. 2014. Evolution of reddit: from the front page of the internet to a self-referential community?. In Proceedings of the 23rd international conference on world wide web. ACM, 517-522.

[22] Richard Skarbez, Frederick P Brooks Jr, and Mary C Whitton. 2018. A survey of presence and related concepts. ACM Computing Surveys (CSUR) 50, 6 (2018), 96.

[23] Michael Steurer, Christoph Trattner, and Frank Kappe. 2012. Success factors of events in virtual worlds a case study in second life. In 2012 11th Annual Workshop on Network and Systems Support for Games (NetGames). IEEE, 1-2.

[24] Marsha White and Steve M Dorman. 2001. Receiving social support online: implications for health education. Health education research 16, 6 (2001), 693-707.

[25] Nick Yee and Jeremy N Bailenson. 2008. A method for longitudinal behavioral data collection in Second Life. Presence: Teleoperators and Virtual Environments 17, 6 (2008), 594-596.

[26] Xi Zhang, Patricia Ordóñez de Pablos, Xiaojiong Wang, Weiguang Wang, Yongqiang Sun, and Jinghuai She. 2014. Understanding the usersâĂŹ continuous adoption of 3D social virtual world in China: A comparative case study. Computers in Human Behavior 35 (2014), 578-585.

[27] Frederick J Zimmerman and Dimitri A Christakis. 2005. ChildrenâĂŹs television viewing and cognitive outcomes: a longitudinal analysis of national data. Archives of Pediatrics \& Adolescent Medicine 159, 7 (2005), 619-625. 